\title{
A Review for the Current Advancements in 5G Technology
}

\author{
Ahmed Najah Jabbar \\ Department of Electrical Engineering, College of Engineering, University of Babylon. \\ Ahmed_Aljafari@yahoo.com \\ Ibrahim A. Murdas \\ Department of Electrical Engineering, College of Engineering, University of Babylon. \\ dr.ibrahim-ba@yahoo.com
}

\begin{abstract}
The fifth generation technology or in short (5G technology) is the recent technology that is meant to break the data limits barrier. It promises very high data rates that will provide the user with enough bandwidth to conduct a real time HD telephone conversation. It will also provide the infrastructure for the IoT (Internet of Things) that will be dedicated for electronic governments, electronic healthcare centers, social media networks, full-scale data sharing, and remote controlling for sensitive governmental facilities. This paper is intended as survey for the current developments and technologies available for the coming 5G mobile technology. It discusses the ideas, the preparations, the developments, the standards under discussion and the potentials for this technology. In addition, this work takes into consideration the challenges and the difficulties facing this new coming technology and the plans laid ahead for the futuristic mobile networks.
\end{abstract}

Keywords: Fifth generation (5G), IoT, New technology in communication, 5G groups.

\section{Introduction:}

The current era has a distinctive mark that can be sensed through the extensive exchange of data. The users are now sharing every moment or feeling throughout the world. This massive transfer of data represents a formidable challenge for the engineers and the companies that built their portfolio and revenues on providing such service. The internet now represents a home for billions of people that talk to each other all over the globe. These vast conversations should be held with utmost accuracy and confidentiality. The entire burden will fall on the backbone of the networks and should be robust enough to handle such barrage of information. The networks were designed to carry and handle moderate loads of data. The sudden jumps in the technology and microelectronics made the dreams come true. The people are now exchanging their every thought and whisper. These advancements in the micro and nanotechnology and electronic made available for every person multiple smart devices that are considered more than just a phone. They are now the daily companion and assistant that connect us to the others on the other side of the world.

The data exchanged needed to be with high quality and real time. They should not be prone to frequent pixilation or disconnections. These criteria are the base and the essence 
of the data companies that can define whether that company will continue in that market or lose the race. These companies constructed huge influential economical entities that can press hard on the market and the legislation institution to pass regulations that serve their interests. Their fingerprints can be seen on the accelerated steps and jumps in the field of communication and data exchange protocols.

Until the recent years, the communication channels were able to convey the enormous amounts of dat. Nevertheless, the expectation for the load growth will soon surpass the physical infrastructure ability to with stand the ever-rising pressure. According the Statista site and the ITU recommendations, the load and traffic will rise exponentially as shown in Fig. 1 [1][2].

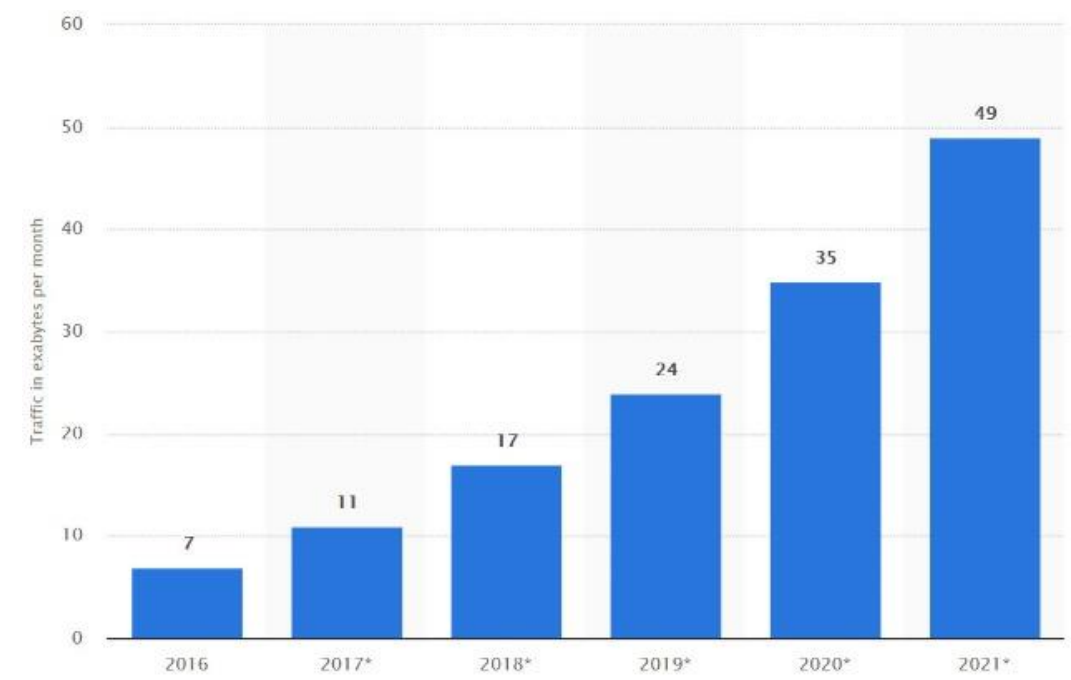

(a)

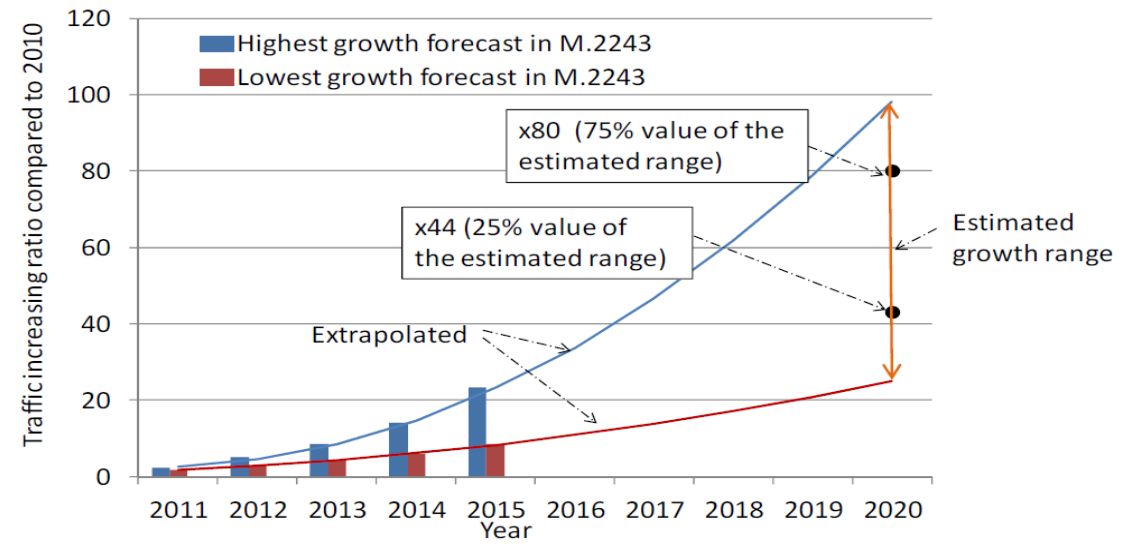

(b)

Fig. 1: The estimated load until 2020-2021, (a) in Exabyte by Statista, (b) Relative increase to 2010 traffic load by ITU.

ITU published another expectation for the load increase until 2030 as shown in Fig. 2 in Exabyte (EB) [3] 
Journal of University of Babylon for Engineering Sciences, Vol. (26), No. (7): 2018.

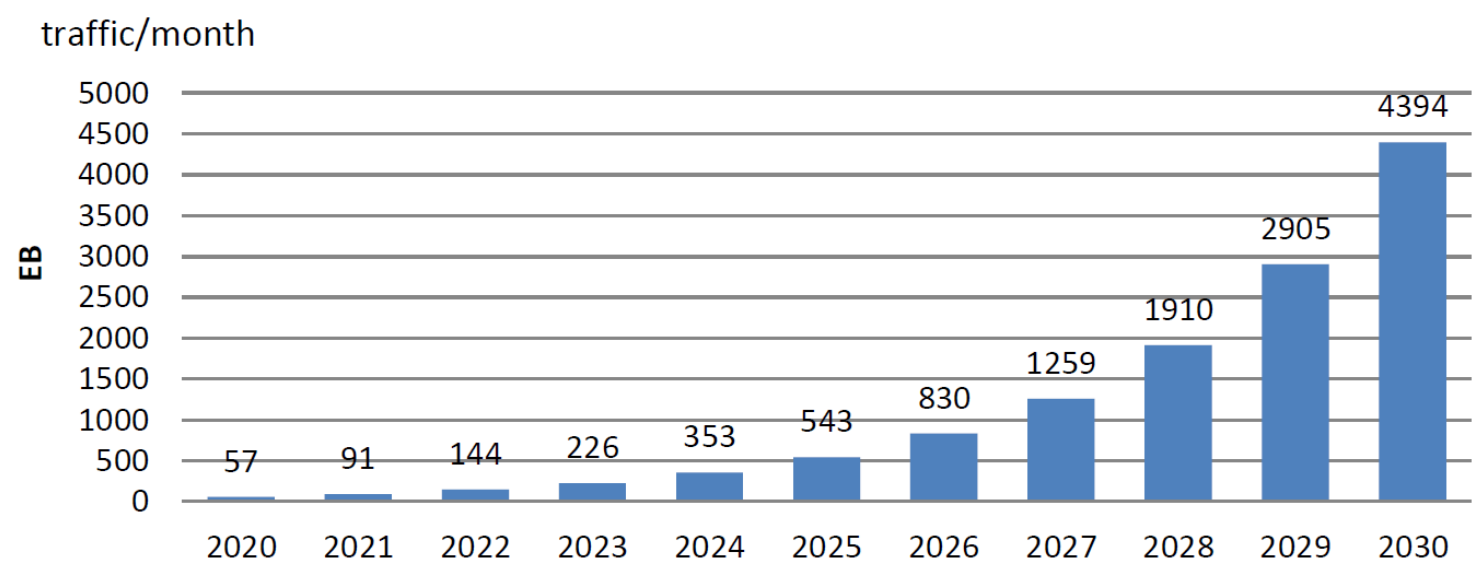

Fig. 2: the estimated load until 2030 by ITU

When comparing Fig. 2 with Fig. 1, we can see the amount of challenge facing the future of communications and data networks. Therefore, the workers in these fields gathered their efforts in an attempt to establish the proper foundations that can face these futuristic challenges. The solutions came in a series of suggested technologies and techniques that were enrolled under the term $5 G$ Technology.

\section{The 5G Technology}

The 5G technology is a term used for the recommendations and suggestions for upgrading the communication networks beyond the LTE or 4th Generation mobile networks. Until now no international standard were published that put a solid frame to define this technology [3]. Hence, the competition to find such firm standards is still going on till clear lines and pivotal points are reached. The 5G technology is emphasizing its efforts on the spectrum utilization, higher bit rates by minimizing the redundant traffic, utilizing higher frequency spectra, re-structuring the protocol and the networks layers for higher performance, including smart and intelligent technologies within its standards like the cognitive radio, implementing massive MIMO to open tracks for the data as much as possible and increasing the personal devices performance for a better spectrum utilization. Therefore, the academicians and the engineers are working feverishly for finding unique solutions and taking out the networks from the box. The workers around the world assembled themselves in global groups to work as bee hives to find the solutions [4] [5] and [6].

\subsection{Global working Groups}

There are groups around the world that can be classified as small enthusiastic groups that provide solution for particular problems and major groups that invests billions of dollars to put the adequate standards for the upcoming communication technology. This section will focus on the influential groups that are entitled to address the final standards of the 5G technology. These groups are [7] [8] and [9]:

2.1.1 The ITU group: The International Telecommunication Union located at Geneva Switzerland is a group founded by the UN to issue standards in the field of communication and allocate the RF spectrum according to the needs of the market. This group researching 
and anticipating the future demand for the $5 \mathrm{G}$ and give solutions to the pre-imagined communication future. Its preliminary task is to tailor new protocols, networks infrastructures and suggests new technology for best bandwidth utilization. Currently, it is working with the major groups to unify the vision for the $5 \mathrm{G}$ technology.

2.1.2 5G Infrastructure Public Private Partnership (5GPPP): This group is one of the major groups working to find the practical implementation for the coming $5 \mathrm{G}$ technology. It is like the 3GPPP and considered as the frontrunner in the field of 5G technology. It houses many subgroups specialized at a certain field in the $5 \mathrm{G}$ technology. These subgroups are:

i. METIS-II EU: The Mobile and wireless communications Enablers for Twenty-twenty (2020) Information Society-II is a European group that is its main vision is to plot the physical structure for the $5 \mathrm{G}$ networks. An international communication companies Ericsson, Telecom Italia, Orange, Deutsche Telecom and a variety of U.S. and European universities are considered as main resources to accomplish their vision regarding $5 \mathrm{G}$.

ii. 5G NORMA: The 5G NORMA stands for 5G Novel Radio Multiservice adaptive network Architecture. The main task for this group is to establish a flexible end to end user architecture. Its main consideration is to develop the necessary software that is resilient enough to ensure proper distribution for the $5 \mathrm{G}$ data traffic.

iii. mmMAGIC: This group is interested in re allocating the RF spectrum for more efficient high rate data transmission. It also explores higher frequencies for potentials that can be invested in the $5 \mathrm{G}$ microwave link. The group is also responsible for designing and developing the necessary microwave devices that will meet it final goal.

2.1.3 Next Generation Mobile Networks (NGMN) Alliance: This alliance includes many influential mobile companies in the US like Verizon and AT\&T. This groups is exploring new patents that will help and aid in the final construction of the 5G. The alliance is also exploring the market potentials for this new coming technology and how to benefit from its services.

2.1.4 IEEE Group: The IEEE group is well known and has a long history in the field of communication and other important fields related to engineering. Its main momentum is based on the contributions of academicians, researchers and visionaries. It has a wellrespected contribution in issuing standards that unify the new emerging technologies around the world.

2.1.5 National Institute of Standards and Technology (NIST): This group is connected to the US department of commerce. This group is interested in the development of massive MIMO, mmWave spectrum and dens data rates [10]. Until now, this groups has no practical contribution in the field of $5 \mathrm{G}$ but it was able to launch a model for the purposes of test for the $5 \mathrm{G}$ adopted by the NGMN.

2.1.6 4G Americas: This group is an advertising group that is working to find major investors and capitals in the 5G technology. Its main working arena is the north and south American continents. 
Journal of University of Babylon for Engineering Sciences, Vol. (26), No. (7): 2018.

2.1.7 FCC: The Federal Communications Commission is a US department that issues regulations and lows to organize the wireless communication activities inside the US. This group will take on the task to distribute the major exchange nodes, how the spectrum should be allocated and the frequencies available.

\subsection{The Planned Features for the 5G Technology}

The next mobile generation should satisfy certain features in order to be ready to operate in the intended massive load environment. The general features are [11] [12] and [13]:

2.2.1 High Speed Links: The workers in the $5 \mathrm{G}$ are planning to establish very highspeed links that are capable of handling huge loads that may reach $1 \mathrm{~Gb}$ per user. The physical structures may include satellite links, millimeteric wave links, dense optical communication and massive MIMOs.

2.2.2 Covering Rural Areas: The new $5 \mathrm{G}$ should be able to cover the rural area that are not covered by the casual ISPs transmission. This is planned to be accomplished by implementing the cognitive radio. The cognitive radio can utilize the available radio spectrum to switch the packets to the nearest ISP with the need for direct connection. The cognitive radio will increase the spectrum efficiency by allowing the licensed users to share the channel with the unlicensed user when it is idle.

2.2.3 High QoS in condensed places: The $5 \mathrm{G}$ provide a high QoS especially in the areas where the users are generating huge traffic. This will decrease the backlog load and the requirements for metadata channels.

2.2.4 Smarter Handovers: The 5G should provide a better hand over by increasing the intelligence of the ground stations.

2.2.5 Relinquishing the Hierarchy: One of the limitations of the generations before the $5 \mathrm{G}(1 \mathrm{G}$ to $4 \mathrm{G})$ is the need for hierarchical system to pass the data from the source to the destination. This will increase the latency and reduces the QoS. Adding to that, the system will be prone to failure and the loss of huge amount of data. Therefore, the next technology will include a new term that is the Flat IP. In this new protocol, all the devices will contribute in the switching and relaying the data from point to point. This will ensure that the reduction in the cost of establishing networks, the distribution of the loads among the users, the alternative paths are available even when the main channel is down and the network can evolve when more users are joining the crowd. The flat IP network is planned to look like Fig. 3. As it can be seen form Fig. 3, the next generation networks are simpler and high the potential for better chance to be upgraded. 


\section{Today's Telecom Networks}

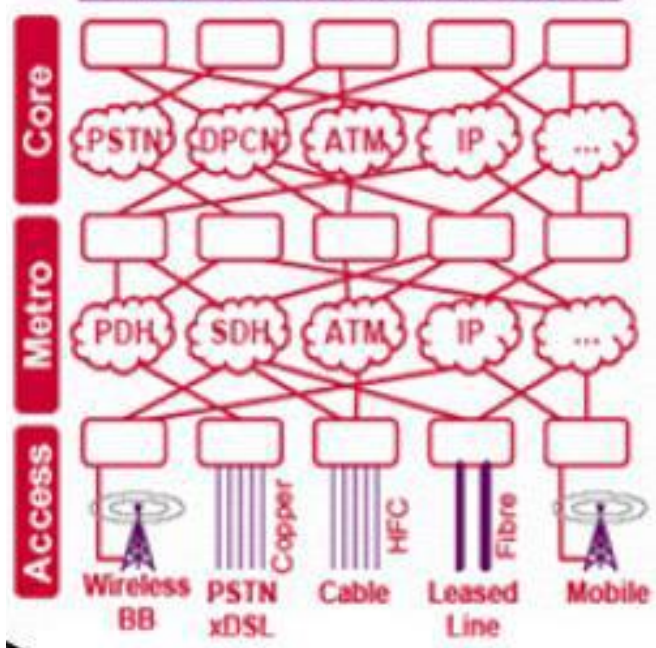

Fig. 3: A comparison between the hieratical and flat IP networks

2.2.6 White Spectrum Efficient Use: The next networks will utilize the white spectrum dedicated for earlier TV and radio broadcast. This utilization will solve the problem of RFspectrum congestion and introduce new band allocations. These new bands will provide higher freedom for the network to evolve and transfer higher loads. The white spectrum is expected to be utilized using the cognitive radio systems.

\section{The Transmission Techniques Available to 5G Technology}

The 4G technology implemented the OFDM as its main modulation technique. It was preferred over the CDMA used by the $3 \mathrm{G}$ because it has a better immunity to Inter Symbol Interference (ISI). Nevertheless, the OFDM seems to be affected by the motion of the mobile network due to the Doppler Effect [14] [17]. In addition, the OFDM wastes about $9 \%$ of its spectrum due to the use of the cyclic prefix to mitigate the channel nonlinearity. Therefore, the OFDM is no longer a candidate for the next mobile networks. Although the OFDM in its current technique is not adequate, but it is the foundation for derived techniques that have a better performance and higher bandwidth utilization. These techniques are:

\subsection{Filter Band Multi Carrier (FBMC)}

The FBMC block diagram is shown in Fig. 4.

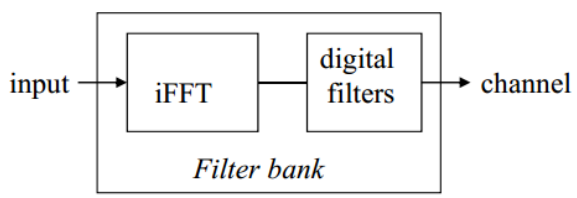

Fig. 4: the FBMC block diagram

As it can be seen from Fig. 4, a digital filter bank is added after the IFFT stage in order to concentrate the signal's Power Spectral Density (PSD) in a confined space. The 
PSD represents the average of the energy amount contained in the signal over a very long period of time in the Fourier domain as show in Eq. 1:

$$
S_{x}(f)=\lim _{T \rightarrow \infty} E\left\{\frac{1}{2 T}\left|\int_{-T}^{T} x(t) e^{-j 2 \pi f t} d t\right|^{2}\right\}
$$

\section{Where:}

$x(t):$ is the signal in time.

$T$ : the averaging time period.

Using the autocorrelation in the frequency domain, we have:

$$
S_{x}(f)=\int_{-T}^{T} R_{x}(\tau) e^{-j 2 \pi f t} d t
$$

And

$$
R_{x}(\tau)=E\{x(t) x *(t+\tau)\}
$$

The PSD is an important measure that shows how the power is distributed at each frequency component.

This concentration yields a better performance for the system and less affected by the noise or the nonlinearity. Hence, the FBMC will provide a better performance with less redundancy that is required by the OFDM used by the current technology.

\subsection{Universal filter multi carrier (UFMC)}

The UFMC is a generalized FBMC where the filtration is acting on a group of subcarriers rather than a single carrier. Figure 5 shows the block diagram for the suggested UFMC [19]

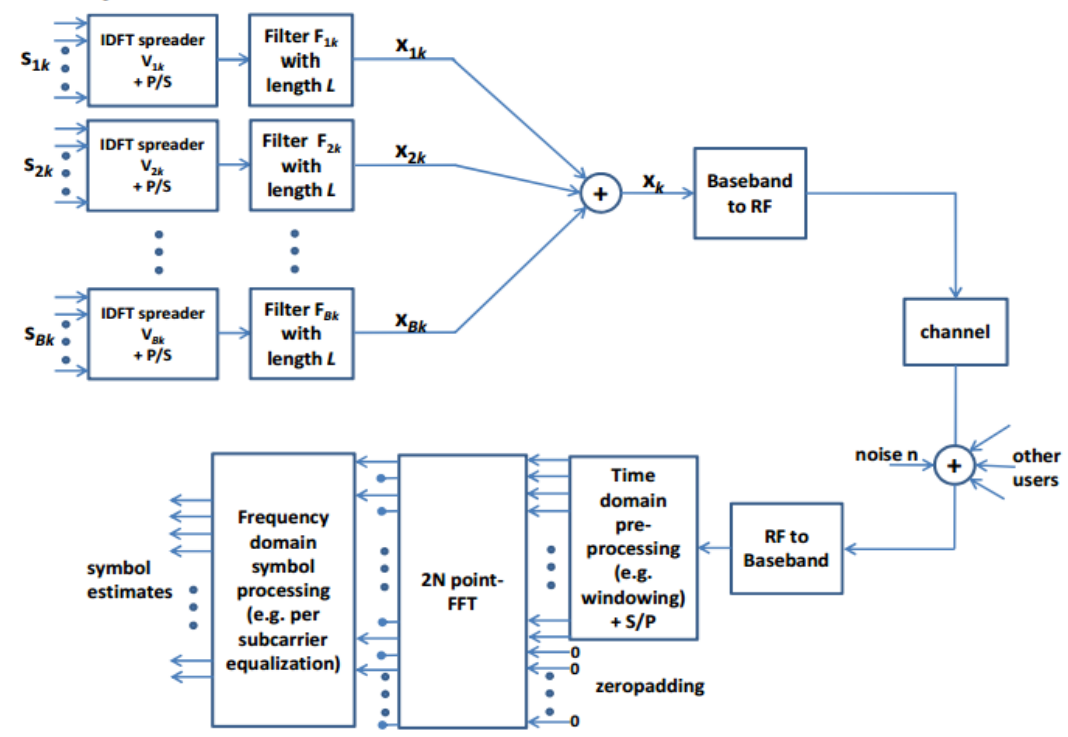

Fig. 5: The UFMC Block Diagram 
This arrangement combines few subcarriers in a group and pass this group through a short band filter. The performance of UFMC can be notice in the burst noise environments. The UFMC has an improved PSD and less sidelobes. Therefore, it is one of the candidates for data modulation techniques.

\subsection{Generalized frequency domain multiplexing (GFDM)}

This technique does not depend on the orthogonalization principle implemented by the OFDM and its derivatives. It rather uses a time space mutiplexing pulsating techniques to transmit the data. This allows to reduce the PSD expansion along with the Peak Average Power Ration (PAPR). Figure 6 shows the block diagram of the GFDM.
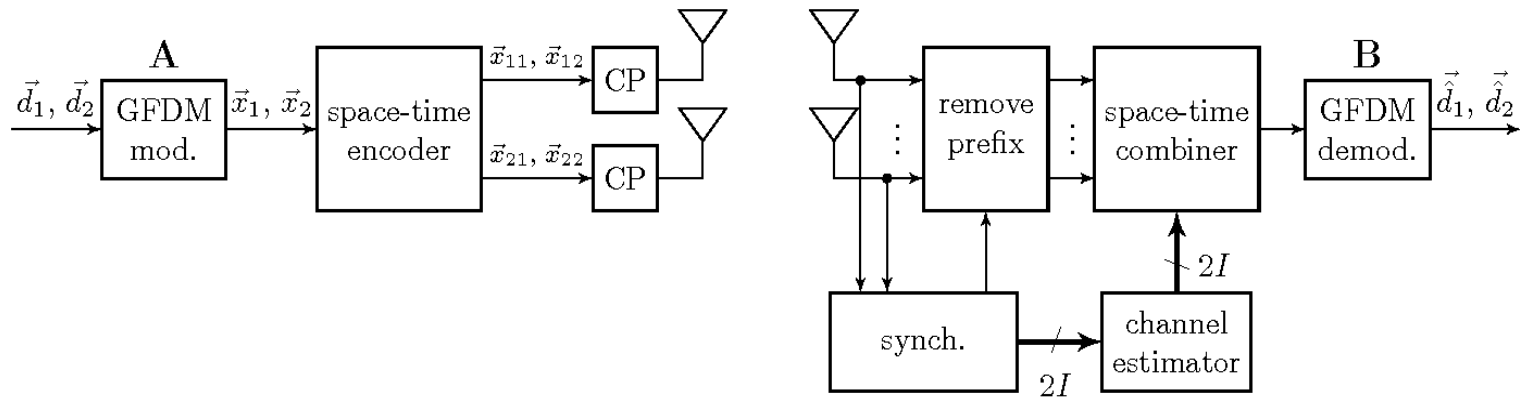

Fig. 6: The GFDM Block Diagram

\subsection{Faster Than Nyquist Rate}

This is a more direct approach for increasing data rate. The data pulses are sent in a very high speed from the source to the destination as shown in Fig. 7. This scheme will cause the lack of orthogonality, increase in ISI and higher sensitivity to the fading in the channel. However, this scheme assumes enough circuitry at the end point that can recover the damaged data.
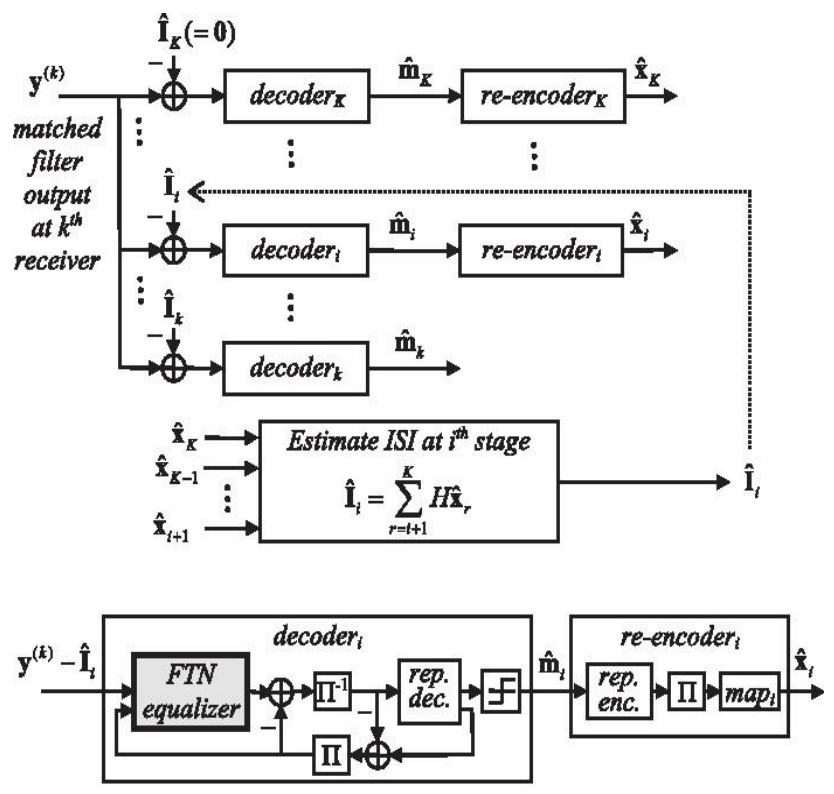

Fig. 7: Faster Than Nyquist Rate Block Diagram 


\subsection{Single-Carrier Modulation (SCM)}

The SCM is a rising contender to replace the multiple access schemes in the next technology, see Fig. 8. The SCM implements the old modulation techniques when each symbol is treated individually rather than grouping the symbols in one bundle. Despite the SCM is an old fashion scheme but it seems adequate and ready to carry it responsibilities in the face of the upcoming challenges.

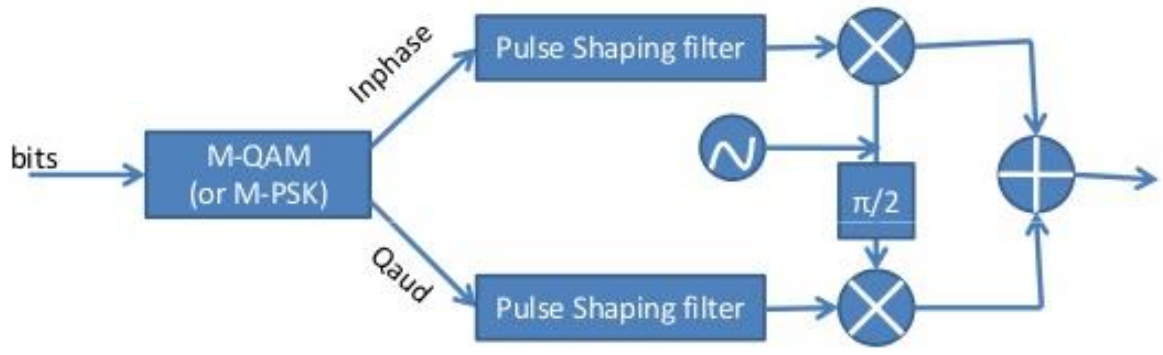

Fig. 8: The SCM Block Diagram

\section{Challenges Facing the 5G Networks}

The planned networks for the 5G is shown in Fig. 9. Such a huge diverse networks imposes so many challenges on the development and designing groups around the world. Therefore, these challenges are left open for the researchers to find proper solutions for them. Below a list for the most pressing issues facing the 5G [19] [20]

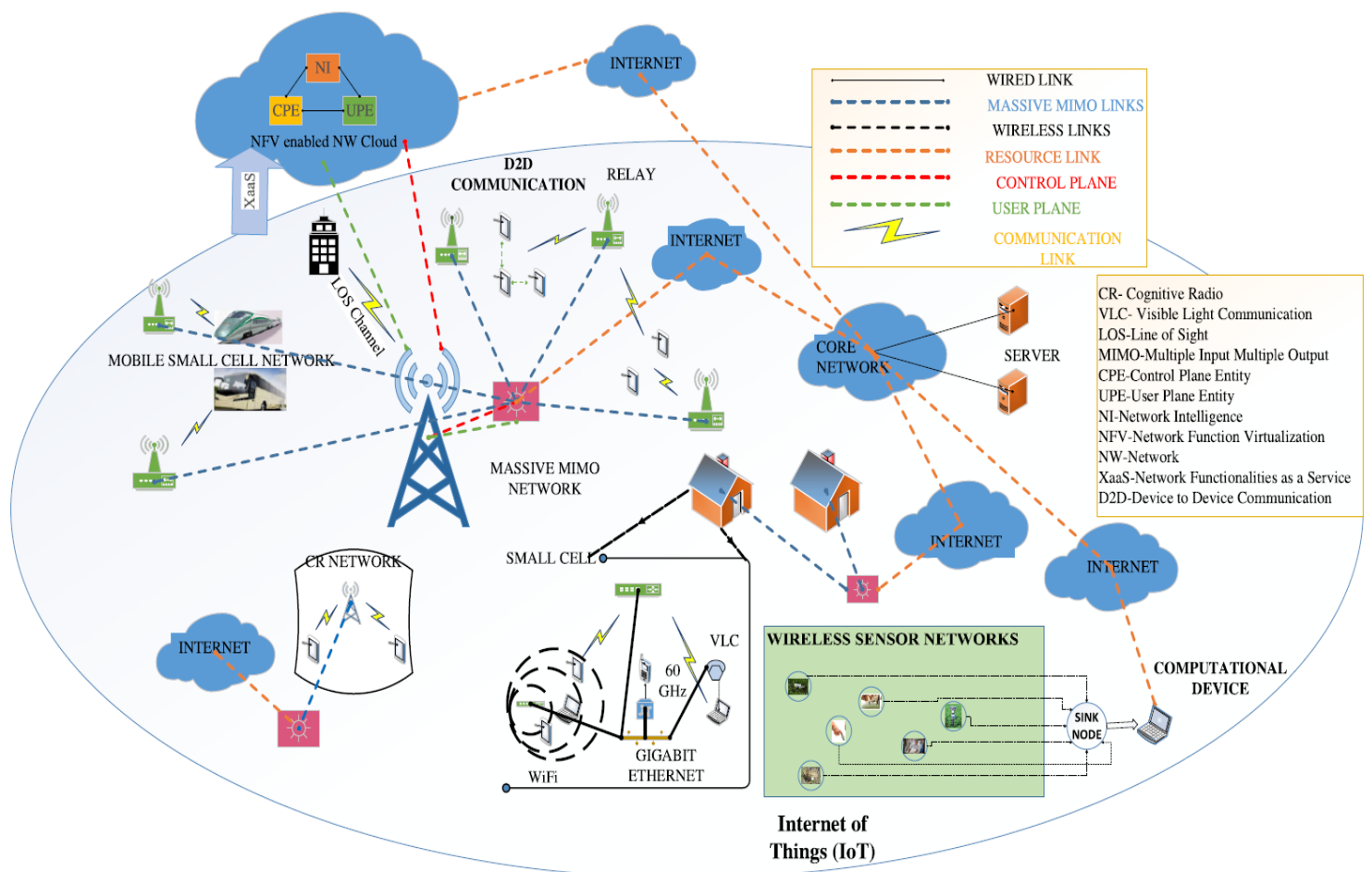

Fig. 9: The Futuristic Vision about the 5G Network 


\subsection{The IoT and the Maximum connections}

The IoT is a growing concept that is trying to allow the users to upload their data to the cloud where the data are put in a pool for sharing. The start for the IoT was to store the user's data and photos but it grows to include various services like traffic monitoring, emedicine, e-governments and e-education and marketing. These widely spread institutions with high traffic need to connect to each other to exchange data. The $3 \mathrm{G}$ and $4 \mathrm{G}$ limits the number of connections per user in order to stabilize the traffic load. The plans for the $5 \mathrm{G}$ is to set no limit for the connections. Therefore, highly advanced protocols are needed to organize and distribute the load without overwhelming certain nodes over others.

\subsection{The amount of Generated Load}

The main purpose for the $5 \mathrm{G}$ development is to provide faster and higher bandwidths for larger amounts of data. As seen in Figs. 1 and 2 the expected load is anticipated to rise exponentially measured by the Exabyte. Therefore, managing such huge data represents a challenge to the $5 \mathrm{G}$ networks. A technique adopted by the $4 \mathrm{G}$ is to consider the voice as an application rather than a data requires separate switching layer. This reduces the complexity and the amount of the data needed to be handled.

\subsection{The Cost versus the Capacity}

The upcoming networks should ensure that the increase in the network capacity and infrastructure should not come with large cost. This will ensure the networks are flexible and easy to install where it is needed. The high prices and complexity will limit the spread the $5 \mathrm{G}$ networks around the world.

\subsection{Simple Efficient Physical Infrastructure}

The deployment for the $5 \mathrm{G}$ network in rural or new areas needed to be easy and efficient. If sensitive, complex and high cost equipment are needed every time the $5 \mathrm{G}$ is deployed then these difficulties will hinder the spread of the new coming networks.

\subsection{Security, Real Time Applications and Sensitive Data Exchange}

The 5G networks is planned to replace the conventional networks and provide alluring opportunity for the sensitive institutions to participate in the 5G. Some of these institutions demands high level of security and certain QoS to ensure real time control or data exchange. Putting all the data in one pool, as suggested by the flat IP, means that the data will be exposed to perpetrators and hacker to change or modify or steal these sensitive information. Therefore, the $5 \mathrm{G}$ should ensure an impenetrable firewalls and security along with the full secrecy for these data. Compromising these two opposites are not an easy task therefore, new intelligent anonymous defenses are required to protect valuable data.

\subsection{Virtual Reality and Augmented Reality}

The virtual reality is now a common widely spread technique used for architectural designs, training, playing games or teaching. The next step is the augmented reality were smells, touch and other sensory data will be available for the users. This new technology 
will require an unimaginable bandwidths to convey such accessory data. With the increase and spread of the augmented reality usage, the $5 \mathrm{G}$ networks need to cope with the demands of this technology.

\subsection{The M2M Challenge}

The automobiles are now connected to the internet or to be planned to be connected. Each car is considered as a machine that require to connect using the network to exchange the necessary data. The cars are built to be smarter faster with services needed by the travelers. The constant motion and the opened doors for the cars to be upgraded into smart machines are wide open. Adding to that, the number of cars are increasing around the world with the increase in population, decrease in average car prices and the necessary need for a car to travel inside the cities. These M2M links are to be handled carefully with precision because they might endanger individuals lives.

\subsection{The D2D Challenge}

The number of devices are increasing rapidly around the world. Each smart devices is considered as the daily companion and partner of the person. These mobile are far from being just a devices to make phone calls because they are housing the memories, the photos, the secrets and bank accounts. The devices are to be linked to each other without the need for the backbone of the network in order to reduce the traffic and complexity of the network. Therefore, novel protocols and schemes are needed to achieve this goal.

Table 1 summarizes the differences and technical specification among the mobile generations from $1 \mathrm{G}$ to the promised $5 \mathrm{G}$ technology.

Table (1): A Comparison among Mobile Technologies for Different Generations

\begin{tabular}{|c|c|c|c|c|c|}
\hline $\begin{array}{c}\text { Mobile } \\
\text { Technology }\end{array}$ & $1 \mathrm{G}$ & $2 \mathrm{G}$ & $3 \mathrm{G}$ & $4 \mathrm{G}$ & $5 \mathrm{G}$ \\
\hline $\begin{array}{l}\text { Design/Laun } \\
\text { ch Date }\end{array}$ & $1970 / 1981$ & 1980/1991 & $1990 / 2001$ & $2000 / 2010$ & $2020 / 2030$ \\
\hline Services & Analog voice & $\begin{array}{l}\text { Supports } \\
\text { Digitized } \\
\text { sound, } \\
\text { provides } \\
\text { short } \\
\text { message } \\
\text { service } \\
\end{array}$ & $\begin{array}{c}\text { Increased } \\
\text { transmission } \\
\text { capacity to } 2 \mathrm{Mbit}\end{array}$ & $\begin{array}{l}\text { Completely } \\
\text { IP oriented, } \\
\text { Multimedia } \\
\text { services, } \\
\text { Capacity } 100 \\
\text { Mbits to } 1 \\
\text { Gbits. } \\
\end{array}$ & $\begin{array}{l}\text { More than } 1 \\
\text { Gbits }\end{array}$ \\
\hline Standards & $\begin{array}{c}\text { NMT, ETACS, } \\
\text { AMPS. }\end{array}$ & $\begin{array}{l}\text { TDMA,CD } \\
\text { MA,GSM }\end{array}$ & $\begin{array}{l}\text { WCDMA, } \\
\text { CDMA-2000, } \\
\text { EDGE }\end{array}$ & LTE, WiFi & $\begin{array}{l}\text { World Wide } \\
\text { Wireless Web } \\
\text { (wwww) }\end{array}$ \\
\hline Frequency & $\begin{array}{c}\text { Analog signal } \\
(30 \mathrm{KHz})\end{array}$ & $\begin{array}{l}1.8 \mathrm{GHz} \\
\text { (digital) }\end{array}$ & $1.6-2.0 \mathrm{GHz}$ & $2-8 \mathrm{GHz}$ & $3-300 \mathrm{GHz}$ \\
\hline $\begin{array}{c}\text { Characteristi } \\
\mathrm{c}\end{array}$ & $\begin{array}{l}\text { First wireless } \\
\text { communication }\end{array}$ & Digital & $\begin{array}{c}\text { Digital } \\
\text { broadband, } \\
\text { increased speed }\end{array}$ & $\begin{array}{l}\text { High speed, } \\
\text { all IP }\end{array}$ & Unknown \\
\hline Bandwidth & $150 / 900 \mathrm{MHz}$ & $900 \mathrm{MHz}$ & $100 \mathrm{MHz}$ & $100 \mathrm{MHz}$ & $\begin{array}{c}\text { 1000x BW pr } \\
\text { unit area }\end{array}$ \\
\hline
\end{tabular}


Journal of University of Babylon for Engineering Sciences, Vol. (26), No. (7): 2018.

\begin{tabular}{|c|c|c|c|c|c|}
\hline Multiplexing & FDMA & $\begin{array}{c}\text { TDMA, } \\
\text { CDMA }\end{array}$ & CDMA & OFDM & Unspecified \\
\hline $\begin{array}{c}\text { Core } \\
\text { Network }\end{array}$ & PSTN & PSTN & Packet network & Internet & $\begin{array}{c}\text { Probably Flat } \\
\text { IP }\end{array}$ \\
\hline Handoff & Horizontal & Horizontal & Horizontal & $\begin{array}{c}\text { Horizontal } \\
\text { and vertical }\end{array}$ & $\begin{array}{c}\text { Horizontal and } \\
\text { vertical }\end{array}$ \\
\hline
\end{tabular}

\section{Conclusions}

This paper shows that the next step in the future in the communication world is inevitable. The constant and increasing demands for e-services and the wide spread of smart devices made the ground fertile for the growth of the data exchange. The expected traffic is expected to multiply many times in comparison with the current traffic load. Therefore, proactive measures should be taken and well planned before the zero hour.

These measures are faced with enormous challenges that made their task uneasy. Therefore, the worldwide mobilization for the brilliant minds and research institutions is necessary to step over the imminent challenges.

The challenges come in different shapes and levels and can be categorized as physical and software component. The physical components are trying to find new highly efficient modulation schemes, high bitrate devices with low power consumption, relocating the RFspectrum by devising new antenna structures and materials. The software challenges come from the angle were the devices should have higher intelligence, more self-organized, higher security and a better utilization for the bandwidth.

The $5 \mathrm{G}$ is not a well defined technology nor a term but it refers to every new innovative technique or suggestion that will contribute in the solution of the massive traffic management and distribution. 
Journal of University of Babylon for Engineering Sciences, Vol. (26), No. (7): 2018.

\section{References:}

[1] Can be found at: https://www.statista.com/statistics/271405/global-mobile-data-trafficforecast/

[2] Future spectrum requirements estimate for terrestrial IMT. Report ITU-R M.2290-0. p. 16; 2014.

[3] IMT traffic estimates for the years 2020 to 2030 IMT. Report M.2370-0; 2015. p. 1014.

[4] Roh, W. et al., "Millimeter-Wave Beamforming as an Enabling Technology for 5G Cellular Communications: Theoretical Feasibility and Prototype Results", IEEE Communications Magazine, pp. 106-113, 2014.

[5] Jameel, F. et. al., "Massive MIMO: A Survey of Recent Advances, Research Issues and Future Directions", International Symposium on Recent Advances in Electrical Engineering (RAEE), 2017.

[6] Bangerter B., "Networks and Devices for the 5G Era", IEEE Communications Magazine, pp. 90-96, 2014.

[7] can be founded at: https://www.fiercewireless.com/special-report/how-itu-5gpppngmn-and-others-will-create-standard-for-5g

[8] Kumar A. and Gupta M., "A review on activities of fifth generation mobile communication system", Alexandria Engineering Journal xxx, $\mathrm{xxx}-\mathrm{xxx}$, in Press, Springer, 2017.

[9] Mitra R. N. and Agrawal D. P., "5G mobile technology: A survey”, ICT Express 1, Elsevier, pp. 132-137, 2015.

[10] Can be fount at: https://www.nist.gov/ctl/5g-mmwave-channel-model-alliance

[11] Ancansa G. et. al., "Spectrum Considerations for 5G Mobile Communication Systems", Procedia Computer Science 104, Elsevier, pp. 509 - 516, 2017.

[12] Ezhilarasan E. and Dinakaran M., "A Review on mobile technologies: 3G, 4G and 5G", Second International Conference on Recent Trends and Challenges in Computational Models, pp. 369-373, 2017.

[13] Shahzaad B. and Azam M. A, "Hierarchical and Flat-Based Hybrid Naming Scheme in Content-Centric Networks of Things", IEEE Internet of Things Journal, Vol. 5, Issue: 2, pp. 1070 - 1080. April 2018.

[14] Bahai A. and Saltzberg B., Multi-Carrier Digital Communications Theory and Applications of OFDM, Kluwer Academic Publishers, N.Y., USA, 2002.

[15] Prasad R., OFDM for Wireless Communications Systems, Artech House, USA, 2004.

[16] Li Y. and Stuber G., Orthogonal Frequency Division Multiplexing for Wireless Communications, Springer, USA, 2006. 
Journal of University of Babylon for Engineering Sciences, Vol. (26), No. (7): 2018.

[17] Schulze H. and Luders C., Theory and Applications of OFDM and CDMA Wideband Wireless Communications, John Wiley, USA, 2005.

[18] Duan S., Yu X., and Wang R., "Performance Analysis on Filter Parameters and Subbands Distribution of Universal Filtered Multi-Carrier", Springer Wireless Personal Communications, Vol. 95, Issue 3, pp 2359-2375, 2017.

[19] Fang D., Qian Y., and Qingyang Hu R., "Security for 5G Mobile Wireless Networks", IEEE Access, Vol. 6, pp. 4850 - 4874, 2017.

[20] Ten 5G Challenges for Engineers to Overcome, can be found at: http://pages.anritsuemearesponse.com/rs/937-UWP-683/images/10-5Gchallenges.pdf?mkt_tok=3RkMMJWWfF9wsRonvqvNZKXonjHpfsX66ugoXKKg3 $\underline{\text { 8431UFwdcjKPmjr1YIFScN0aPyQAgobGp5I5FEBSbfYSbdwt6MFWA\%3D\%3D. }}$ 
Journal of University of Babylon for Engineering Sciences, Vol. (26), No. (7): 2018.

\section{ورقة مراجعة في تكنلوجيا الجيل الخامس من الناحية التقنية و التطويرات في هذا المجال}

$$
\text { أحمد نجاح جبار }
$$

قسم الهندسة الكهربائية، كلية المندسة، جامعة بابل

Ahmed_Aljafari@yahoo.com

$$
\text { إبر اهيم عبد الرحمن }
$$

قسم العندسة الكهربائية، كلبة العهنسة، جامعة بابل

\section{dr.ibrahim-ba@yahoo.com}

\section{الخلاصة}

الجيل الخامس للاتصالات أو ما يشار اليه باختصار (5G) يمثل النطور الجديد في عالم الاتصالات و الذي

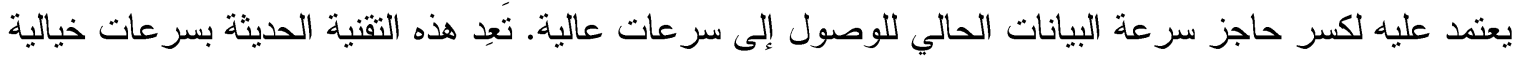
ستمكن المستخدمين من إجر اء مكالمات صورية ذات دقة عالية جدا وبوقت حقيقي دون تقطيعات. كما وستوفر هذه التهات

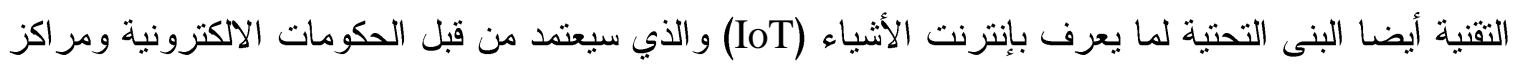

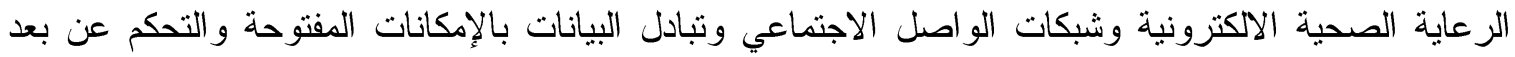

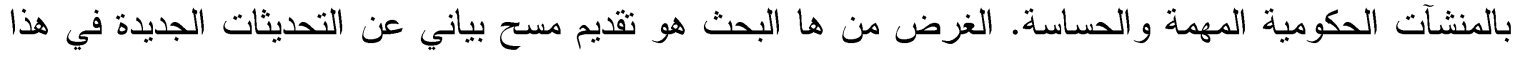

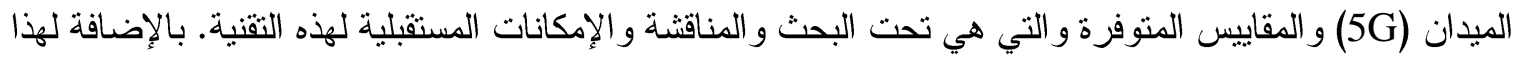
ينطرق البحث لموضوع التحديات والصعوبات التي تواجه هذه التقنية الحديثة والخطط الموضوعة لمستقبل

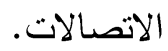

الكلمات المفتاحية: - الجيل الخام، انترنيت الأثشياء، النقنيات الحديثة في الاتصالات، مجاميع الجيل الخامس. 